\title{
Transient Dynamical-Thermal-Optical System Modeling and Simulation
}

\author{
Luzia Hahn ${ }^{1, *}$ and Peter Eberhard ${ }^{1, * *}$ \\ ${ }^{1}$ Institute of Engineering and Computational Mechanics, Pfaffenwaldring 9, 70569 Stuttgart, Germany
}

\begin{abstract}
In this work, methods and procedures are investigated for the holistic simulation of the dynamicalthermal behavior of high-performance optics like lithography objectives. Flexible multibody systems in combination with model order reduction methods, finite element thermal analysis and optical system analyses are used for transient simulations of the dynamical-thermal behavior of optical systems at low computational cost.
\end{abstract}

\section{Introduction}

High resolution optical systems like astronomical telescopes or lithographic objectives consist of precise mirrors or lenses. The optical elements are mounted with high accuracy and they are very sensitive with respect to mechanical vibrations and temperature changes.

In the waver production, scanning systems use lithography objectives to project structures for a certain time onto the wafers. During the operation time, even small mechanical disturbances of the mirrors or lenses or temperature changes in the optical elements can lead to aberrated images. Sources of the vibrations can be, e.g., from influences of the wafer motion system which lead to excitations at the objective frame. The ever-growing demand of high throughput in the scanning systems demands a rise in the power of the used lasers [1]. This leads to heating of the lenses or mirrors and can therefore result in thermal aberrations. The dynamical-thermal behavior of optical systems can be described by a combination of rigid body motion, small deformations, the related stresses, material property changes, and the temperature distribution.

In order to investigate the overall dynamical-thermaloptical behavior, multi-disciplinary methods and software tools with suitable interfaces have to be used and combined. Here, a method is proposed for simulating the performance of dynamical-thermal-optical systems. Thereby, time-dependent deformations due to mechanical excitations and changes in the refraction index due to temperature gradients within the optical elements are considered. An exemplary system simulation is presented for a single lens system, shown in Fig. 1.

\section{Modeling}

Dynamical-thermal-optical system analysis includes the modeling and simulation of the movement of a mechanical system, the simulation of the heat conduction in the

\footnotetext{
*e-mail: luzia.hahn@itm.uni-stuttgart.de

**e-mail: peter.eberhard@itm.uni-stuttgart.de
}

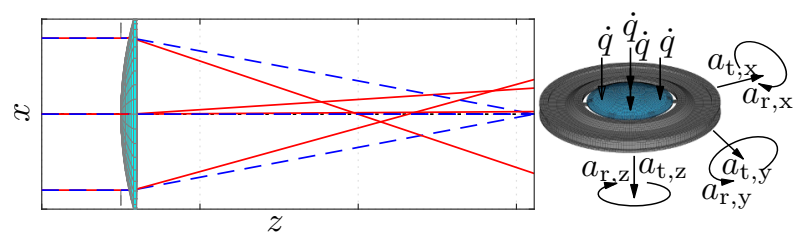

Figure 1. Single Lens optical system with all excitations (right) and the raypaths of the ideal system (blue, dashed) and the dynamical and thermal excited system (red).

elements and the consideration of the associated effects in the optical system simulation. Mechanical excitations lead to deformations and thus to mechanical stresses in the optical elements. Thermal changes lead to material property changes, thermal stresses, and temperature-induced displacements. A generalized workflow using cooperating software packages according to Fig. 2 can be used for holistic dynamical-thermal-optical system simulations. Therby, the mechanical deformations and stresses are considered. The thermal effects are only considered with the thermally induced refraction index changes during the optical simulation.

The first step in the mechanical modeling is, to model each optical sensitive component separately as an elastic body using a finite element (FE) software. The extraction of the node coordinates and the system matrices leads to the equations of motion which enable the calculation of the motion of each elastic body as a function of time. Additionally, the mode shapes are extracted and can be used in a model order reduction in order to reduce the number of degrees of freedom and to speed up the mechanical simulations. Afterwards, the elastic bodies and the remaining system components, modeled as rigid bodies, are assembled in a flexible multibody system [2]. The last step of the mechanical modeling is a numerical time integration which results in the movements and deformations of the dynamical-optical system dependent on time. The dynamical-optical system modeling is as also shown in [3] and [4]. 


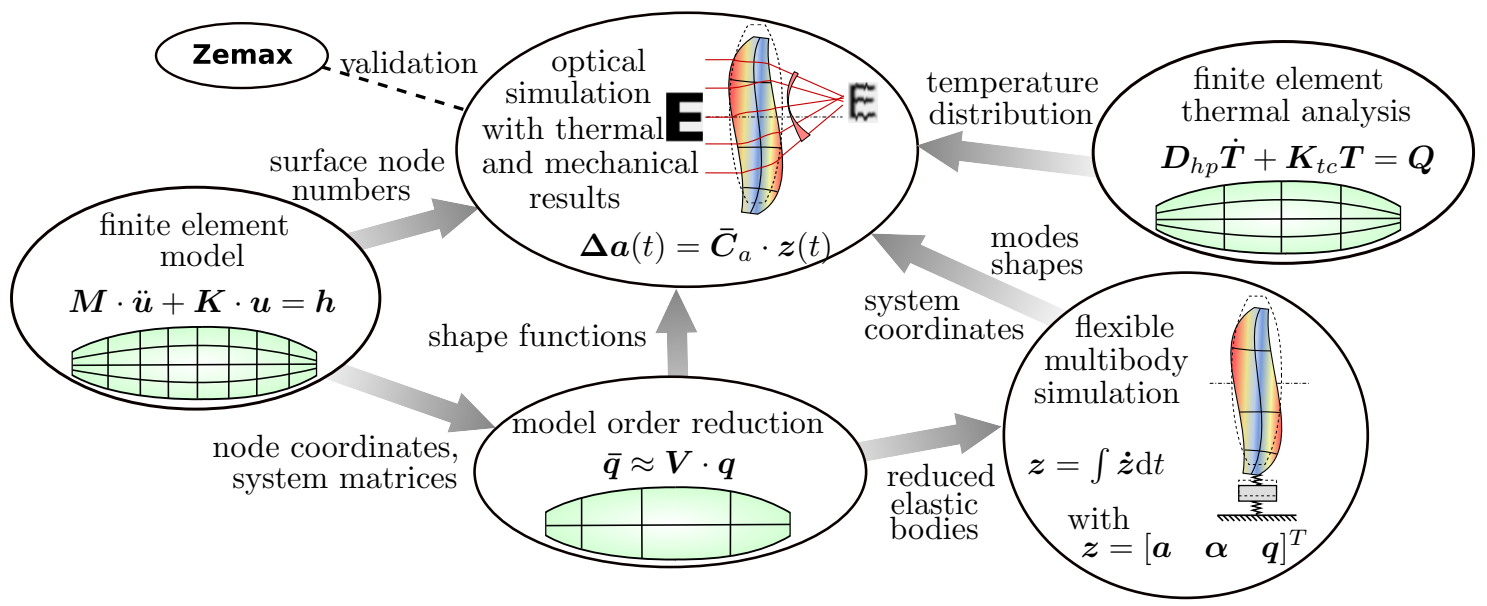

Figure 2. Workflow of the described dynamical-optical system simulation.

In order to consider the thermal effects in the system, an FE thermal analysis is applied to the thermal sensitive components. This results in the time dependent temperatures at each node of the thermal FE mesh.

After the mechanical and the thermal time simulations, sequential ray tracing which considers the thermal and the mechanical results is performed. Therefore, the rigid body motion of each optical element is transformed from the absolute description to a relative description and during the ray tracing the mode shapes, representing the deformations of the reduced bodies, are approximated accurately by polynomial functions, e.g., using a set of Zernike polynomials. Besides that, the displacements of the mechanical mesh nodes are interpolated to the thermal mesh using inverse distance weighting. The changes in the refraction index due to the temperature changes are calculated [5] and either described analytically, e.g. with cylindrical polynomials and evaluated or interpolated in the ray tracing. The optical lenses which are subject to mechanical stresses, a non uniform temperature distribution, or both have a gradient in the refraction index (GRIN). For the ray tracing through these lenses, a fourth order Runge-Kutta method is used. The rays are traces stepwise through the GRIN lenses and in each spatial step the changes in the refraction index due to stress and temperature are calculated and considered. For the optical simulation considering the mechanical and thermal effects, the program OM-Sim is developed. Besides the ray tracing, it can provide the associated wavefront aberrations and an image simulation.

\section{Numerical Example and Conclusion}

As an example of the performance of an integrated dynamical-thermal-optical system simulation a single lens system is presented in Fig. 1. The dashed lines show exemplary four ray paths for the ideal system. The lens is dynamically excited with a predefined movement in all space directions and an analytical, radial temperature gradient around the points of impact of the rays to the lens is applied. Stresses are not considered in this example. The resulting ray paths for the mechanical and thermal excited system are presented in Fig. 1 in red. The mechanical deformation and the thermal gradient was scaled during the ray tracing such that the aberration effects get visible. Due to the radial temperature gradient, the focal length decreases as it can be seen in pink in Fig. 3. Due to the mechanical deformations the focus point is shifted away from the optical axis, as shown in Fig. 3 with the green dashed rays. So, the dynamical and thermal effects influence the imaging performance of the optical system and both should be considered in a holistic optical system simulation.

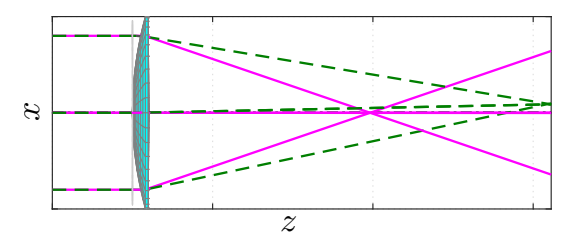

Figure 3. Raypaths of the thermal excited system (pink) and the dynamical excited system (green, dashed).

\section{References}

[1] L. Zhao, L. Dong, X. Yu, P. Li, P. Qiao,Active lens for thermal aberration compensation in lithography lens, Appl. Opt. 57(29), 8654-8663 (2018)

[2] R. Schwertassek, O. Wallrapp, Dynamik flexibler Mehrkörpersysteme. (in German) (Vieweg, Braunschweig, 1999)

[3] N. Wengert, Gekoppelte dynamisch-optische Simulation von Hochleistungsobjektiven. (in German) (Dissertation, Schriften aus dem Institut für Technische und Numerische Mechanik der Universität Stuttgart 40, Shaker Verlag, Aachen, 2015)

[4] J. Störkle, Dynamic simulation and control of optical systems (Dissertation, Schriften aus dem Institut für Technische und Numerische Mechanik der Universität Stuttgart 58, Shaker Verlag, Aachen 2018)

[5] N.N., Temperature Coefficient of the Refractive Index, SCHOTT Technical Information TIE-19, 2 (2016). 